\title{
Venous Thromboembolism Involving Internal Jugular, Subclavian, Axillar and Brachial Veins in a Patient with Multiple Thrombophilic Defects and Malignancy
}

\author{
Pierpaolo Di Micco,", Giuseppe Vito Viggiano ${ }^{2}$, Maria Rosaria Diadema ${ }^{3}$ and Alferio Niglio ${ }^{2}$ \\ ${ }^{1}$ Internal Medicine, Fatebenefratelli Hospital of Naples, Naples, Italy \\ ${ }^{2}$ Internal Medicine Division, Second University of Naples, Naples, Italy \\ ${ }^{3}$ Oncology Division, Second University of Naples, Naples, Italy
}

\begin{abstract}
Cancer and its related therapies are acquired thrombophilic risk factors. Patients with a malignancy can suffer a thrombotic complication such as deep venous thrombosis (DVT) in the lower extremities with a possible following pulmonary embolism. Recently, an increased association between upper extremity DVT and malignancy has been observed and several risk factors were identified. However, few data are available for the association between upper extremity DVT and inherited thrombophilia in oncological patients. Here we report a case of upper extremity DVT complicated by fatal pulmonary embolism in an oncological patient receiving chemotherapy and carrying 2 heterozygous genetic thrombophilic risk factors.
\end{abstract}

Keywords: Upper limb deep vein thrombosis, malignancy, inherited thrombophilia, factor V Leiden, prothrombin A20210G.

\section{INTRODUCTION}

Venous thromboembolism (VTE) is a multifactorial disease which may appear as deep venous thrombosis of lower extremities (LEDVT) or more rarely of the upper extremities (UEDVT) with possible life-threatening complications such as pulmonary embolism (PE) [1].

UEDVTs account for approximately $4 \%$ of all VTE events, and its incidence has recently increased [2]. This increase may be due to the development of more accurate diagnostic methods, such as ultrasonography with colorDoppler flow [3-5].

Risk factors for VTE include an inherited thrombotic predisposition and acquired trigger factors $[1,6,7]$. A clear association between thrombophilic conditions and VTE has been identified in particular for LEDVT, while the same association is still matter of discussion for UEDVT $[6,7]$. Yet, recently an acquired condition associated with thrombophilia, heparin-induced thrombocytopenia, has been reported as a trigger for UEDVT besides LEDVT [8]. However, recent studies report a possible association between thrombophilic condition and UEDVT and also UEDVT and PE recurrence [9].

Commonly, studies that investigate thrombophilia do not also analyse molecular aspects of thrombophilia.

We report a case of a man affected by malignancy who experienced UEDVT and fatal PE associated with the presence of acquired and genetic thrombophilic risk factors.

*Address correspondence to this author at the Internal Medicine, Fatebenefratelli Hospital of Naples, Via San Giacomo dei Capri 69, I-80131, Naples, Italy; E-mail: pdimicco@libero.it

\section{CASE PRESENTATION}

We present the case of a 62 year old male smoker with lung cancer (adenocarcinoma; T2N2M1 stage) and several metastatic lesions in the liver and brain. Because surgery was not possible he started chemotherapy with carboplatin and vincristine. The patient did not recall any previous thrombotic disorder. To facilitate drug administration and further therapeutic support a central venous lines was placed in the right subclavian vein. Thromboprophylaxis with enoxaparin, $4000 \mathrm{U}$ daily, was started to prevent VTE. After the second chemotherapeutic cycle he suddenly developed fever and severe pain in the right upper limb associated with dyspnea. Clinical examination revealed a swollen right upper limb associated with cutaneous venous ectasia and impaired movement. An ultrasound scan with color-Doppler revealed a deep venous thrombosis of the right extremity venous axis involving the internal jugular and subclavian, axillary and brachial veins. We performed arterial gas analysis that showed respiratory alkalosis with hypoxia and hypocapnia (pH: 7.49, $\mathrm{PaO}_{2}, 88 \mathrm{mmHg}, \mathrm{PaCO}_{2}, 24 \mathrm{mmHg}$ ). A thoracic helical CT scan revealed a sub-massive pulmonary embolism with involvement of sub-segmentary vessels. A partial occlusion of the central venous catheter was also seen. Treatment with unfractionated heparin $(30000 \mathrm{U}$ of i.v. sodium heparin) was started. Heparin administration was monitored with an activated partial thromboplastin ratio range of 1.52.5. Antibiotic prophylaxis was also started to prevent septic complications (i.e. ceftriaxone $1 \mathrm{~g}$ daily for 2 days). Because the severity of embolic manifestations we chose not to remove the indwelling catheter.

After 3 days of treatment a thrombophilic screening was performed and revealed normal values of protein $\mathrm{C}$, protein $\mathrm{S}$ and antithrombin (i.e. $70 \%, 65 \%$ and $81 \%$, respectively), 
normal values of anticardiolipin antibodies $\operatorname{IgG}$ and $\operatorname{IgM}$, normal prothrombin time with an INR of 0.9. Homocysteine was normal (i.e. $12 \mu \mathrm{mol} / \mathrm{L}$ ). An altered activated protein $\mathrm{C}$ resistance (tested according to the method described by Dahlback) [10] was revealed and a genetic test to detect the presence of factor $\mathrm{V}$ Leiden suggested further investigation (see below). We report all thrombophilic tests in Table 1.

Table 1. Molecular Thrombophilic Tests of Described Patients

\begin{tabular}{|l|c|c|}
\hline & Patient & $\begin{array}{c}\text { Normal } \\
\text { Values }\end{array}$ \\
\hline \hline PT (INR) & 0.97 & $0.8-1.2$ \\
\hline aPTT (ratio) & 0.85 & $0.8-1.2$ \\
\hline Fibrinogen (mg/dl) & 340 & $240-420$ \\
\hline AT III (\%) & 82 & $80-120$ \\
\hline APCR (Dahlback method) & 1.89 & $>2.00$ \\
\hline PC (\%) & 84 & $62-120$ \\
\hline PS (\%) & 97 & $65-120$ \\
\hline Anticardiolipin antibodies IgM (UM/l) & 3 & $<10$ \\
\hline Anticardiolipin antibodies IgG (UG/l) & 4 & $<10$ \\
\hline Lupus anticoagulant & Absent & Absent \\
\hline Factor V Leiden gene variant & Heterozigosity & Wyld type \\
\hline Prothrombin A20210G gene variant & Heterozigosity & Wyld type \\
\hline MTHFR C67T gene variant & Wyld type & Wyld type \\
\hline
\end{tabular}

PT: protrhombin time; aPTT: activated partial thromboplastin time; AT: antithrombin; APCR: activated protein C resistance; PC: protein C; PS: protein S; MTHFR: methylene-tetra-hyfolate reductase.

Thrombophilic screening was completed by genetic testing to detect known variants such as polymorphisms in prothrombin (A20210G), factor V (Leiden variant, FVL) and methylenetetrahydrofolate reductase (MTHFR C677T). DNA was extracted using "NUCLEON BACC2" kit (Amersham, Germany) and PCR amplification was carried out with specific primers and Light Cycler apparatus (Roche Diagnostic, Italy) by "melting" curve analysis. The patient was a combined heterozygous carrier of the FVL and the prothrombin A20210G gene variants.

After 10 days of i.v. antithrombotic treatment the patient showed a further decrease of respiratory performance with a significant increase of pulmonary artery pressure and a worsening shock index with right ventricular dysfunction. He died 5 days later.

\section{DISCUSSION}

Malignancy is the most common cause of cause of acquired thrombophilia and it is commonly associated with VTE during the natural history of the disease [1]. Inherited thrombophilia is also associated to VTE including early onset [7]. On the other hand, UEDVT was considered very rare until the last decades, when an increased incidence has been recorded possibly because of an improved knowledge of risk factors and better technological support with ultrasound scans $[1,5]$. Few data are available on the incidence of UEDVT in oncological patients also carrying inherited thrombophilia. Some authors, recently described UEDVT in such patients in association with inherited thrombophilia and during secondary thromboprophylaxis $[11,12]$. The role of acquired risk factors for triggering venous thrombosis such as an indwelling venous catheter in oncological patients with or without inherited thrombophilia has been recently underlined $[11,12]$.

This case is interesting because the patient had double thrombophilic gene variants (i.e. heterozygous for FVL and prothrombin A20210G) without a history of previous thrombotic events. VTE occurred in spite of primary thromboprophylaxis with low molecular weight heparin (LMWH). The patient had several thrombotic risk factors (inherited and acquired) that could trigger a thrombotic event (i.e. genetic thrombophilic variants, malignancy, chemotherapy, central venous line). The role of molecular thrombophilia in the occurrence of VTE has been investigated in the last years and underlined a specific role in cases of early onset and the presence of further first degree relative affected by VTE. Moreover, this trend to develop VTE for carriers of molecular thrombophilia seems to be increased in presence of further thrombotic risk factors. The efficiency of thrombophilia testing in patients with VTE has been recently analysed and showed that patients affected by any type of VTE carrying thrombophilia were 10 years younger compared with patients without thrombophilia independently of additional risk factors [13]. Moreover, in this particular case, besides the presence of several acquired thrombotic risk factors (e.g. cancer, chemotherapy and venous catheter) we detected 2 thrombophilic variants (factor V Leiden and the G20210A prothrombin mutation) resulting in an increased risk of venous thrombosis [14].

The outcome of this kind of patients may be difficult because several complications may appear such as PE andlor sepsis. Our case raises questions such as the appearance of VTE during primary thromboprophylaxis with an appropriate dose of LMWH. Thrombosis occurred with multiple thrombotic risk factors including 2 different inherited thrombophilic defects. So for this kind of subject (i.e. oncological patients that need a central venous line) we suggest screening for inherited thrombophilia in order to establish appropriate thromboprophylaxis.

\section{CONSENT}

Written informed consent was obtained from the patient for publication of the manuscript.

\section{REFERENCES}

[1] Di Micco P, Amitrano M, Niglio A, Fontanella A. Molecular and clinical conditions associated with venous thromboembolism in oncological patients. Exp Oncol 2006; 28: 245-7.

[2] Bolgiano EB, Foxwell MM, Browne BJ, et al. Deep venous thrombosis of the upper extremity: diagnosis and treatment. J Emerg Med 1990; 8: 85-91.

[3] Monreal M, Munoz FJ, Rosa V, et al. Upper extremity DVT in oncological patients: analysis of risk factors. Data from the RIETE registry. Exp Oncol 2006; 28: $194-7$.

[4] Di Micco P, Di Fiore R, Quaranta S, et al. Upper limb deep vein thrombosis: update on risk factors in oncological patients. Cancer Ther 2007; 5: 391-4.

[5] Kearon C, Ginsberg JS, Hirsh J. The role of venous ultrasonography in the diagnosis of suspected deep venous thrombosis and pulmonary embolism. Ann Intern Med 1998; 129: 1044-9.

[6] Martinelli I, Cattaneo M, Panzeri D, et al. Risk factors for deep venous thrombosis of the upper extremities. Ann Intern Med 1997; 126: 707-11. 
[7] Martinelli I. Risk factors in venous thromboembolism. Thromb Haemost 2001; 86: 395-403.

[8] Warkentin TE, Cook DJ, Flinterman LE, et al. Current perspective of venous thrombosis in the upper extremity. J Thromb Haemost 2008; 6 [Epub ahead of print].

[9] Martinelli I, Battaglioli T, Bucciarelli P, Passamonti SM, Mannucci PM. Risk factors and recurrence of primary deep vein thrombosis of the upper extremities. Circulation 2004; 110: 566-70.

[10] Dahlback B. The discovery of activated protein $\mathrm{C}$ resistance. J Thromb Haemost 2003; 1: 3-9.

[11] Muñoz FJ, Mismetti P, Poggio R, et al.; RIETE Investigators. Clinical outcome of patients with upper-extremity deep vein thrombosis: results from the RIETE registry. Chest 2008; 133: 1438.
[12] Monreal M, Munoz FJ, Rosa V, et al. Upper extremity DVT in oncological patients: analysis of risk factors. Data from the RIETE registry. Exp Oncol 2006; 28: 245-7.

[13] Roldan V, Lecumberri R, Sanchez Munoz-Torrero JF, Vicente V, Rocha E, Brenner B; Monreal for the RIETE Investigators. Efficiency of thrombophilia screening in patients with venous thromboembolism. findings from the RIETE registry. Thromb Res 2009 (in press).

[14] De Stefano V, Martinelli I, Mannucci PM, et al. The risk of recurrent deep venous thrombosis among heterozygous carriers of both factor V Leiden and the G20210A prothrombin mutation. N Engl J Med 1999; 341: 801-6.

Received: October 20, 2008

(C) Di Micco et al.; Licensee Bentham Open.

This is an open access article licensed under the terms of the Creative Commons Attribution Non-Commercial License (http://creativecommons.org/licenses/by$\mathrm{nc} / 3.0 /$ ) which permits unrestricted, non-commercial use, distribution and reproduction in any medium, provided the work is properly cited. 